\title{
Kidney Function Decline in the Elderly: Impact of Lipoprotein-Associated Phospholipase $\mathbf{A}_{2}$
}

\author{
Carmen A. Peralta ${ }^{a, b}$ Ronit Katz ${ }^{c}$ Michael Shlipak ${ }^{a, b}$ Ruth Dubin ${ }^{b}$ \\ Ian DeBoer ${ }^{c}$ Nancy Jenny ${ }^{d}$ Annette Fitzpatrick ${ }^{c}$ Carol Koro $^{e}$ \\ Bryan Kestenbaum $^{c}$ Joachim Ix ${ }^{f}$ Mark Sarnak $^{g}$ Mary Cushman ${ }^{d}$ \\ aSan Francisco VA Medical Center, and ' University of California, San Francisco, San Francisco, Calif., \\ 'University of Washington, Seattle, Wash., 'University of Vermont College of Medicine, Burlington, Vt.,

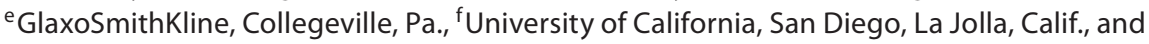 \\ gTufts University School of Medicine, Boston, Mass., USA
}

\section{Key Words}

Chronic kidney disease $\cdot$ Elderly $\cdot$ Estimated GFR $\cdot$ Kidney

decline $\cdot$ Lipoprotein-associated phospholipase $\mathrm{A}_{2}$

\begin{abstract}
Background: Whether lipoprotein-associated phospholipase $A_{2}\left(L p-P L A_{2}\right)$ levels are associated with kidney function decline has not been well studied. Methods: We investigated associations of $\mathrm{Lp}-\mathrm{PLA} \mathrm{A}_{2}$ antigen and activity with kidney function decline and rapid decline over 5.7 years in the Cardiovascular Health Study $(n=4,359)$. We estimated kidney function by cystatin C (eGFRcys) in repeated measures, and defined rapid decline as $\geq 3 \mathrm{ml} / \mathrm{min} / 1.73 \mathrm{~m}^{2}$ per year. We stratified by baseline preserved GFR ( $\left.\geq 60 \mathrm{ml} / \mathrm{min} / 1.73 \mathrm{~m}^{2}\right)$. Results: Mean age was $72 \pm 5$ years. Average eGFRcys decline was $-1.79 \mathrm{ml} / \mathrm{min} / 1.73 \mathrm{~m}^{2}(\mathrm{SD}=2.60)$ per year. Among persons with preserved GFR, compared to the lowest quartile of Lp-PLA $A_{2}$ antigen, eGFRcys decline was faster among persons in the second, $\beta-0.31(95 \% \mathrm{Cl}-0.52,-0.10)$, third -0.19 $(-0.41,0.02)$ and fourth quartiles $-0.26(-0.48,-0.04)$ after full adjustment. Persons in the highest quartile of $\mathrm{Lp}-\mathrm{PLA}_{2}$ antigen had increased odds of rapid decline $1.34(1.03,1.75)$, com-
\end{abstract}

pared to the lowest. There was no significant association between levels of $L p-P L A_{2}$ activity and eGFRcys decline or rapid decline. Associations were not statistically significant among persons with low eGFR $\left(<60 \mathrm{ml} / \mathrm{min} / 1.73 \mathrm{~m}^{2}\right)$ at baseline. Conclusion: Higher levels of $L p-P L A_{2}$ antigen but not activity were significantly associated with faster rates of kidney function decline. These findings may suggest a novel vascular pathway for kidney disease progression.

Copyright $\odot 2011$ S. Karger AG, Basel

\section{Introduction}

Chronic kidney disease (CKD) is associated with increased risk for cardiovascular disease (CVD), $[1,2]$ even at early stages of kidney dysfunction [3]. In addition, clinical and subclinical CVDs are independent predictors of kidney function decline [4]. Proposed mechanisms to explain these associations have included increased inflammation or oxidative stress associated with vascular damage. However, the associations of inflammatory markers and kidney function are modest, and the direction of the association remains unclear [5-7].

\section{KARGER}

(C) 2011 S. Karger AG, Basel

Fax +41613061234 E-Mail karger@karger.ch www.karger.com www.karger.com/ajn
Carmen A. Peralta, MD, MAS

4150 Clement Street, 111A1

San Francisco, CA 94121 (USA)

Tel. +1 415221 4810, ext. 2093

E-Mail carmenalicia.peralta@ucsf.edu 
Lipoprotein-associated phospholipase $\mathrm{A}_{2}$ (Lp-PLA $)$ has been independently associated with CVD in several studies [8-11]. Lp-PLA ${ }_{2}$ is bound to low-density lipoprotein (LDL) cholesterol in the circulation, it is highly expressed in coronary plaques, and it is thought to have proinflammatory properties. Lp-PLA ${ }_{2}$ is also thought to promote endothelial dysfunction and increased inflammation within arterial walls [12-15]. Since endothelial dysfunction and increased arterial stiffness $[16,17]$ have been associated with kidney dysfunction, Lp-PLA 2 may be a plausible pathway associated with kidney dysfunction. The association between Lp-PLA 2 and kidney function decline has not been evaluated.

Understanding whether Lp-PLA 2 is associated with kidney function decline may help elucidate pathways that lead to kidney disease. Therefore, we studied the association of Lp-PLA $A_{2}$ antigen (mass) and activity with kidney function decline among participants in the Cardiovascular Health Study (CHS). We hypothesized that Lp-PLA would be associated with kidney function decline, independent of serum lipid concentrations.

\section{Subjects and Methods}

\section{Subjects}

We included participants from the CHS, a community-based longitudinal study designed to understand risk factors for development and progression of CVD. Details on design and recruitment have been previously published [18]. Briefly, CHS recruited adults who were 65 years of age or older and living in the community by using Medicare eligibility lists. CHS recruited in four sites: Forsyth County, N.C.; Sacramento County, Calif.; Washington County, Md., and Pittsburgh, Pa. Participants were excluded if they were not expected to remain in the current community for 3 years or longer, were receiving treatment for cancer, or were unable to provide informed consent. The initial 5,201 participants were enrolled from January 1989 to June 1990; an additional 687 black participants (with race self-reported) were recruited and enrolled by June 1993. The institutional review boards at all participating centers approved these studies, and all participants gave informed consent.

For these analyses, we included all participants who had a measure of kidney function at baseline and at least one follow-up measure of kidney function and who had measures of Lp-PLA 2 at baseline $(n=4,362)$.

\section{Primary Predictors}

Our two primary predictors of interest were Lp-PLA 2 antigen (mass) and activity. In CHS, phlebotomy was performed after a 12hour fast, and all assays were performed in frozen serum specimens that were stored at $-70^{\circ} \mathrm{C}$. Plasma Lp-PLA ${ }_{2}$ antigen (mass) was measured at the University of Vermont using a commercially available enzyme-linked immunosorbent assay (ELISA; second generation PLAC Test; diaDexus Inc., South San Francisco, Calif., USA). Plas- ma Lp-PLA 2 activity was measured at GlaxoSmithKline (Research Triangle, N.C., USA) using a high-throughput radiometric assay, as previously reported. The interassay coefficients of variation were $6.3 \%$ for antigen and $7.5 \%$ for activity [10]. In CHS, the correlation coefficient between antigen (mass) and activity was 0.51 .

\section{Primary Outcomes}

Kidney function measures included serum creatinine and cystatin C. Cystatin C was measured by means of a particle-enhanced immunonephelometric assay (N Latex Cystatin C; Dade Behring) with a nephelometer (BNII; Dade Behring). Serum creatinine was measured by a colorimetric method (Ektachem 700; Eastman Kodak). Kidney function was measured at baseline and at year 3 and 7 visits. Overall, 2,539 persons had 3 measures of kidney function and 1,823 had 2 measures. We estimated the glomerular filtration rate (eGFR) with the use of the CKD-EPI creatinine equation (eGFRcreat) and the CKD-EPI cystatin C equation (eGFRcys) without demographic coefficients: eGFRcys $=76.7 \times$ cys $^{-1.19}$. Both formulae were developed from the pooling of several cohorts with GFR measured from iothalamate clearance $[19,20]$.

The primary outcome of interest was kidney function decline, assessed by cystatin C using repeated measures of eGFRcys. A secondary outcome of interest was rapid kidney function decline, defined as eGFRcys decline of $\geq 3 \mathrm{ml} / \mathrm{min} /$ year, as has been defined in previous studies showing the association of this definition with adverse outcomes [21].

For these two outcomes, we stratified a priori by whether persons had preserved GFR or established CKD, defined as eGFRcreat $<60 \mathrm{ml} / \mathrm{min} / 1.73 \mathrm{~m}^{2}$. In particular, we chose to define $\mathrm{CKD}$ at baseline based on creatinine because it is still the clinical standard [22]. We chose to study kidney function decline and rapid decline based on eGFRcys rather than eGFRcreat serial measures for several reasons. First, longitudinal measures of eGFRcys have been shown to better correlate with changes in kidney function by direct GFR measurement [23]. Second, we have reported that eGFRcys is an important predictor of aging success in the elderly [24] and that eGFRcys detects kidney function decline in the elderly better than creatinine across the spectrum of GFR [25]. Third, our group has recently shown that only CKD that is confirmed by eGFRcys $<60 \mathrm{ml} / \mathrm{min} / 1.73 \mathrm{~m}^{2}$ is associated with increased risk for death, CVD events, heart failure and end-stage renal disease, compared to CKD detected by creatinine alone [26].

\section{Covariates}

All CHS participants underwent a comprehensive examination at each visit. Age, gender, race, and medication use were ascertained by questionnaire at the baseline visit. Diabetes was defined as a self-report of diabetes, the use of insulin or oral hypoglycemic agents or a fasting glucose $\geq 126 \mathrm{mg} / \mathrm{dl}$. Three blood pressure measurements were obtained $5 \mathrm{~min}$ apart in the seated position. The mean of the second two measurements was used for analysis. Prevalent CVD was defined as having a history of coronary heart disease, heart failure or stroke. High-density lipoprotein (HDL) cholesterol was measured using the Olympus Demand System (Olympus, Lake Success, N.Y., USA). LDL cholesterol was calculated by the Friedewald formula after excluding participants with triglycerides $>400 \mathrm{mg} / \mathrm{dl}$. Details on assays have been previously published [27]. C-reactive protein (CRP) was measured with an ELISA and interleukin-6 (IL-6) was measured by ELISA (R\&D Systems, Minneapolis, Minn., USA). 
Table 1. Baseline characteristics by Lp-PLA 2 antigen

\begin{tabular}{|c|c|c|c|c|}
\hline & Quartile $1(\mathrm{n}=1,136)$ & Quartile $2(\mathrm{n}=1,089)$ & Quartile $3(\mathrm{n}=1,086)$ & Quartile $4(\mathrm{n}=1,048)$ \\
\hline Range of Lp-PLA ${ }_{2}$ antigen, $\mathrm{ng} / \mathrm{ml}$ & $87.08-257.30$ & $257.31-326.67$ & $326.68-404.88$ & $404.89-944.25$ \\
\hline Age, years & $72 \pm 5$ & $72 \pm 5$ & $72 \pm 5$ & $72 \pm 5$ \\
\hline Males & $386(34)$ & $411(38)$ & $474(44)$ & $478(46)$ \\
\hline African-Americans & $261(23)$ & $160(15)$ & $102(9)$ & $61(6)$ \\
\hline Body mass index & $26.8 \pm 5.0$ & $26.7 \pm 4.6$ & $26.7 \pm 4.3$ & $26.5 \pm 4.4$ \\
\hline \multicolumn{5}{|l|}{ Smoking } \\
\hline Never & $557(49)$ & $531(49)$ & $494(46)$ & $461(44)$ \\
\hline Former & $465(41)$ & $453(42)$ & $474(44)$ & $453(43)$ \\
\hline Current & $112(10)$ & $104(10)$ & $117(11)$ & $133(13)$ \\
\hline Hypertension & $476(42)$ & $463(43)$ & $453(42)$ & $447(43)$ \\
\hline Diabetes & $170(15)$ & $157(14)$ & $140(13)$ & $150(14)$ \\
\hline Systolic blood pressure, $\mathrm{mm} \mathrm{Hg}$ & $134 \pm 20$ & $136 \pm 21$ & $135 \pm 21$ & $136 \pm 22$ \\
\hline Diastolic blood pressure, $\mathrm{mm} \mathrm{Hg}$ & $71 \pm 11$ & $71 \pm 11$ & $70 \pm 11$ & $70 \pm 11$ \\
\hline Hypertension medications & $530(47)$ & $484(44)$ & $478(44)$ & $481(46)$ \\
\hline Insulin, IU/ml & $12(9,17)$ & $12(9,17)$ & $13(10,17)$ & $13(10,18)$ \\
\hline Glucose, mg/dl & $109 \pm 34$ & $110 \pm 37$ & $108 \pm 28$ & $109 \pm 30$ \\
\hline LDL cholesterol, mg/dl & $118 \pm 33$ & $128 \pm 34$ & $134 \pm 34$ & $141 \pm 36$ \\
\hline HDL cholesterol, mg/dl & $56 \pm 16$ & $56 \pm 16$ & $54 \pm 15$ & $53 \pm 15$ \\
\hline Triglycerides, mg/dl & $114(87,161)$ & $122(91,160)$ & $126(95,170)$ & $119(95,163)$ \\
\hline Lipid-lowering medications & $89(8)$ & $67(6)$ & $52(5)$ & $34(3)$ \\
\hline $\mathrm{CRP}, \mathrm{mg} / \mathrm{ml}$ & $2.32(1.18,4.36)$ & $2.44(1.15,4.25)$ & $2.37(1.23,3.75)$ & $2.46(1.35,4.32)$ \\
\hline IL-6, pg/ml & $1.54(1.03,2.29)$ & $1.59(1.10,2.43)$ & $1.59(1.12,2.34)$ & $1.62(1.16,2.52)$ \\
\hline Prevalent $\mathrm{CHF}$ & $34(3)$ & $33(3)$ & $23(2)$ & $41(4)$ \\
\hline Prevalent CHD & $173(15)$ & $179(16)$ & $205(19)$ & $208(20)$ \\
\hline eGFR-cysC, $\mathrm{ml} / \mathrm{min} / 1.73 \mathrm{~m}^{2}$ & $83 \pm 18$ & $80 \pm 19$ & $79 \pm 18$ & $76 \pm 19$ \\
\hline eGFR-CKD Epi, $\mathrm{ml} / \mathrm{min} / 1.73 \mathrm{~m}^{2}$ & $76 \pm 17$ & $73 \pm 17$ & $73 \pm 16$ & $72 \pm 17$ \\
\hline
\end{tabular}

Results are presented as mean $\pm \mathrm{SD}$, median (IQR) or numbers with percentages in parentheses. CHF $=\mathrm{Congestive} \mathrm{heart} \mathrm{failure;}$ $\mathrm{CHD}=$ coronary heart disease; eGFR-CKD Epi $=$ CKD-Epi-based eGFR.

\section{Statistical Analyses}

We compared baseline characteristics by quartiles of $\mathrm{Lp}_{\mathrm{PLA}}$ antigen (mass) using $\chi^{2}$ or ANOVA, as appropriate.

We first evaluated the association between Lp-PLA 2 antigen and activity separately with kidney function decline. We used linear mixed models with random intercepts and slopes to estimate and compare linear trends in mean eGFR. This approach takes into account the correlation of observations by subject. Lp-PLA 2 antigen and activity were modeled linearly per standard deviation (SD) and categorically as quartiles. We used nested models with serial adjustment to understand the role of potential confounders. Model 1 adjusted for age, gender, and race. Model 2 adjusted for model 1 plus diabetes, systolic blood pressure, use of antihypertensive medications, LDL cholesterol, HDL cholesterol, use of lipid lowering medications, and prevalent CVD. Finally, we were interested in understanding whether inflammation may play a role in explaining possible associations. Therefore, model 3 added CRP and IL- 6 .

We used multivariate logistic regression to study the association of Lp-PLA 2 antigen and activity with rapid decline. We adjusted in nested models as above.

Earlier literature has suggested different strengths of association between Lp-PLA 2 and cardiovascular outcomes among per- sons in different strata of lipids and CRP $[10,28]$, so we also performed stratified analyses by LDL cholesterol (above and below $130 \mathrm{mg} / \mathrm{dl}$ ), HDL cholesterol (above and below 40 and $50 \mathrm{mg} / \mathrm{dl}$ for men and women, respectively) and CRP (above and below $3 \mathrm{mg} / \mathrm{l}$ ). All analyses were performed using S-Plus (release 8.0; Insightful, Inc., Seattle, Wash., USA) and SPPS (version 16.0.2; SPSS, Inc., Chicago, Ill., USA).

\section{Results}

Among the 4,359 participants in this study, mean age was 72 ( $\pm 5 \mathrm{SD}$ ) years, $13 \%$ were black and $60 \%$ female. Mean eGFRcys at baseline was $80 \mathrm{ml} / \mathrm{min} / 1.73 \mathrm{~m}^{2}(\mathrm{SD}=$ 19) and mean eGFRcreat was $74 \mathrm{ml} / \mathrm{min} / 1.73 \mathrm{~m}^{2}(\mathrm{SD}=$ 17). The mean for Lp-PLA 2 antigen level was $341 \mathrm{ng} / \mathrm{ml}$ $(\mathrm{SD}=118)$ and activity was $39 \mathrm{nmol} / \mathrm{min} / \mathrm{l}(\mathrm{SD}=13)$.

Persons in the highest quartile of Lp-PLA 2 antigen levels had higher baseline levels of LDL cholesterol, lower levels of HDL cholesterol, and were less likely to be on a 
Table 2. Association of Lp-PLA 2 with kidney function decline over 5.7 years among elderly persons by baseline CKD

\begin{tabular}{|c|c|c|c|c|c|c|c|c|}
\hline \multirow[t]{2}{*}{ Models } & \multicolumn{4}{|c|}{ Lp-PLA ${ }_{2}$ antigen $^{\mathrm{a}}$} & \multicolumn{4}{|c|}{ Lp-PLA activity $^{\mathrm{a}}$} \\
\hline & per $\mathrm{SD}=118$ & quartile 2 & quartile 3 & quartile 4 & per $\mathrm{SD}=113$ & quartile 2 & quartile 3 & quartile 4 \\
\hline \multicolumn{9}{|c|}{ eGFRcreat $\geq 60 \mathrm{ml} / \mathrm{min} / 1.73 \mathrm{~m}^{2}$} \\
\hline Subjects & 3,370 & 838 & 833 & 765 & 3,364 & 880 & 803 & 786 \\
\hline Age, sex, race & $\begin{array}{l}-0.10 \\
(-0.18,-0.03)\end{array}$ & $\begin{array}{l}-0.26 \\
(-0.47,-0.06)\end{array}$ & $\begin{array}{l}-0.20 \\
(-0.41,0.001)\end{array}$ & $\begin{array}{l}-0.26 \\
(-0.47,-0.05)\end{array}$ & $\begin{array}{l}-0.05 \\
(-0.12,0.03)\end{array}$ & $\begin{array}{l}0.05 \\
(-0.15,0.26)\end{array}$ & $\begin{array}{l}-0.13 \\
(-0.34,0.08)\end{array}$ & $\begin{array}{l}-0.01 \\
(-0.23,0.20) \\
\end{array}$ \\
\hline+ Risk factors ${ }^{\mathrm{b}}$ & $\begin{array}{l}-0.10 \\
(-0.17,-0.02)\end{array}$ & $\begin{array}{l}-0.28 \\
(-0.49,-0.07)\end{array}$ & $\begin{array}{l}-0.22 \\
(-0.43,-0.01)\end{array}$ & $\begin{array}{l}-0.25 \\
(-0.46,-0.03)\end{array}$ & $\begin{array}{l}-0.04 \\
(-0.12,0.03)\end{array}$ & $\begin{array}{l}0.05 \\
(-0.16,0.26) \\
\end{array}$ & $\begin{array}{l}-0.14 \\
(-0.35,0.08)\end{array}$ & $\begin{array}{l}-0.005 \\
(-0.22,0.21)\end{array}$ \\
\hline + CRP and IL-6 & $\begin{array}{l}-0.10 \\
(-0.18,-0.02)\end{array}$ & $\begin{array}{l}-0.31 \\
(-0.52,-0.10)\end{array}$ & $\begin{array}{l}-0.19 \\
(-0.41,0.02)\end{array}$ & $\begin{array}{l}-0.26 \\
(-0.48,-0.04)\end{array}$ & $\begin{array}{l}-0.04 \\
(-0.12,0.04)\end{array}$ & $\begin{array}{l}0.09 \\
(-0.12,0.31)\end{array}$ & $\begin{array}{l}-0.10 \\
(-0.32,0.12)\end{array}$ & $\begin{array}{l}0.0003 \\
(-0.22,0.22)\end{array}$ \\
\hline \multicolumn{9}{|c|}{ eGFRcreat $<60 \mathrm{ml} / \mathrm{min} / 1.73 \mathrm{~m}^{2}$} \\
\hline Subjects & 989 & 251 & 253 & 283 & 987 & 234 & 249 & 288 \\
\hline Age, sex, race & $\begin{array}{l}-0.01 \\
(-0.31,0.28)\end{array}$ & $\begin{array}{l}0.07 \\
(-0.30,0.44)\end{array}$ & $\begin{array}{l}-0.21 \\
(-0.57,0.16)\end{array}$ & $\begin{array}{l}0.11 \\
(-0.25,0.47)\end{array}$ & $\begin{array}{l}-0.06 \\
(-0.18,0.05)\end{array}$ & $\begin{array}{l}-0.12 \\
(-0.49,0.25)\end{array}$ & $\begin{array}{l}-0.07 \\
(-0.44,0.29)\end{array}$ & $\begin{array}{l}-0.19 \\
(-0.55,0.17) \\
\end{array}$ \\
\hline+ Risk factors ${ }^{\mathrm{b}}$ & $\begin{array}{l}-0.02 \\
(-0.32,0.27)\end{array}$ & $\begin{array}{l}0.05 \\
(-0.32,0.42)\end{array}$ & $\begin{array}{l}-0.23 \\
(-0.60,0.14)\end{array}$ & $\begin{array}{l}0.10 \\
(-0.26,0.47)\end{array}$ & $\begin{array}{l}-0.07 \\
(-0.19,0.05)\end{array}$ & $\begin{array}{l}-0.12 \\
(-0.49,0.25)\end{array}$ & $\begin{array}{l}-0.07 \\
(-0.44,0.30)\end{array}$ & $\begin{array}{l}-0.19 \\
(-0.55,0.17)\end{array}$ \\
\hline + CRP and IL-6 & $\begin{array}{l}-0.02 \\
(-0.37,0.32)\end{array}$ & $\begin{array}{l}0.07 \\
(-0.32,0.45)\end{array}$ & $\begin{array}{l}-0.29 \\
(-0.68,0.10)\end{array}$ & $\begin{array}{l}0.10 \\
(-0.28,0.48)\end{array}$ & $\begin{array}{l}-0.03 \\
(-0.15,0.10)\end{array}$ & $\begin{array}{l}-0.11 \\
(-0.50,0.29)\end{array}$ & $\begin{array}{l}-0.01 \\
(-0.41,0.38)\end{array}$ & $\begin{array}{l}-0.06 \\
(-0.45,0.32)\end{array}$ \\
\hline \multicolumn{9}{|c|}{$\begin{array}{l}\text { Results represent } \beta(95 \% \mathrm{CI}) \mathrm{ml} / \mathrm{min} / 1.73 \mathrm{~m}^{2} \text { per year, except where otherwise indicated. Baseline CKD defined by CKD-Epi eGFR } \geq \text { or }<60 \mathrm{ml} / \\
\mathrm{min} / 1.73 \mathrm{~m}^{2} \text {. Quartile } 1 \text { is the reference group. } \\
{ }^{\text {a } Q u a r t i l e ~ r a n g e s ~ f o r ~ L p-~} \mathrm{PLA}_{2} \text { antigen }(\mathrm{ng} / \mathrm{ml}):<257.31,257.31-326.67,326.68-404.68,>404.68 \text {; quartile ranges for Lp-PLA } 2 \text { activity }(\mathrm{nmol} / \mathrm{min} / \mathrm{ml}) \text { : } \\
<30.41,30.42-37.82,37.83-46.60,>46.60 \text {. } \\
{ }^{\mathrm{b}} \text { Diabetes, systolic blood pressure, antihypertensive medications, LDL and HDL cholesterol, lipid-lowering medications, prevalent coronary heart } \\
\text { disease. }\end{array}$} \\
\hline
\end{tabular}

lipid-lowering agent. Persons in the highest quartile of Lp-PLA ${ }_{2}$ antigen also had lower baseline eGFR by cystatin $\mathrm{C}$ and creatinine (table 1 ).

\section{Lp-PLA $A_{2}$ and Kidney Function Decline}

The average decline in eGFRcys during a median follow up of 5.7 years was $-1.79 \mathrm{ml} / \mathrm{min} / 1.73 \mathrm{~m}^{2}(\mathrm{SD}=2.60)$ per year.

Among persons with eGFRcreat $\geq 60 \mathrm{ml} / \mathrm{min} / 1.73 \mathrm{~m}^{2}$, higher levels of Lp-PLA 2 antigen were significantly associated with faster kidney function decline, and this association was independent of demographics, comorbidities, CRP or IL-6 (table 2). Compared to persons in the lowest quartile of Lp-PLA 2 antigen level, those in quartile 4 had approximately $15 \%$ faster rates of kidney function decline. These associations were not present among persons with eGFRcreat $<60 \mathrm{ml} / \mathrm{min} / 1.73 \mathrm{~m}^{2}$ at baseline (table 2).

In contrast to findings with Lp-PLA ${ }_{2}$ antigen, Lp$\mathrm{PLA}_{2}$ activity level was not significantly associated with kidney function decline (table 2).

\section{Lp-PLA $A_{2}$ and Rapid Kidney Function Decline}

A total of $1,084(25 \%)$ subjects had a rapid decline in kidney function. Among persons with eGFRcreat $\geq 60$ $\mathrm{ml} / \mathrm{min} / 1.73 \mathrm{~m}^{2}$ at baseline, 855 persons had a rapid decline, and 229 persons in the eGFRcreat $<60 \mathrm{ml} / \mathrm{min} /$ $1.73 \mathrm{~m}^{2}$ group. Lp-PLA $\mathrm{P}_{2}$ antigen levels were also independently associated with higher odds of rapid kidney function decline among persons with eGFRcreat $\geq 60 \mathrm{ml} /$ $\mathrm{min} / 1.73 \mathrm{~m}^{2}$ at baseline. Each SD increase in Lp-PLA antigen was associated with $14 \%$ higher odds of rapid decline (table 3). When we categorized Lp-PLA 2 antigen, only the highest quartile was significantly associated with higher odds of rapid decline compared to the lowest quartile (table 3). These associations were not significant among persons with eGFRcreat $<60 \mathrm{ml} / \mathrm{min} / 1.73 \mathrm{~m}^{2}$ at baseline. Lp-PLA ${ }_{2}$ activity was not statistically significantly associated with rapid decline (table 3 ).

\section{Stratified Analyses}

After full adjustment, the association between Lp$\mathrm{PLA}_{2}$ antigen and kidney function decline was not significantly different by LDL or HDL level ( $\mathrm{p}$ value for interaction 0.34 and 0.69 , respectively). Moreover, there was no significant effect modification by CRP on the association between Lp-PLA ${ }_{2}$ antigen and kidney function decline ( $\mathrm{p}$ value for interaction 0.79 ; fig. 1 ). 
Table 3. Association of Lp-PLA 2 with rapid kidney function decline among elderly persons by baseline CKD

\begin{tabular}{|c|c|c|c|c|c|c|c|c|}
\hline \multirow[t]{2}{*}{ Models } & \multicolumn{4}{|c|}{ Lp-PLA $_{2}$ antigen $^{\mathrm{a}}$} & \multicolumn{4}{|c|}{ Lp-PLA ${ }_{2}$ activity $^{\mathrm{a}}$} \\
\hline & per $\mathrm{SD}=118$ & quartile 2 & quartile 3 & quartile 4 & per $\mathrm{SD}=13$ & quartile 2 & quartile 3 & quartile 4 \\
\hline \multicolumn{9}{|c|}{$e G F R \geq 60 \mathrm{ml} / \mathrm{min} / 1.73 \mathrm{~m}^{2}$} \\
\hline Subjects & 3,370 & 838 & 833 & 765 & 3,364 & 880 & 803 & 786 \\
\hline $\begin{array}{l}\text { Age, sex, race, baseline } \\
\text { eGFR-cysC }\end{array}$ & $\begin{array}{l}1.14 \\
(1.04,1.24)\end{array}$ & $\begin{array}{l}1.23 \\
(0.97,1.56)\end{array}$ & $\begin{array}{l}1.04 \\
(0.81,1.34)\end{array}$ & $\begin{array}{l}1.37 \\
(1.06,1.76)\end{array}$ & $\begin{array}{l}1.08 \\
(0.98,1.18)\end{array}$ & $\begin{array}{l}0.96 \\
(0.76,1.22)\end{array}$ & $\begin{array}{l}1.00 \\
(0.78,1.29)\end{array}$ & $\begin{array}{l}1.17 \\
(0.90,1.51)\end{array}$ \\
\hline + Risk factors ${ }^{\mathrm{b}}$ & $\begin{array}{l}1.14 \\
(1.04,1.26)\end{array}$ & $\begin{array}{l}1.23 \\
(0.96,1.56)\end{array}$ & $\begin{array}{l}1.03 \\
(0.80,1.33)\end{array}$ & $\begin{array}{l}1.35 \\
(1.04,1.76)\end{array}$ & $\begin{array}{l}1.05 \\
(0.94,1.17) \\
\end{array}$ & $\begin{array}{l}0.96 \\
(0.75,1.23) \\
\end{array}$ & $\begin{array}{l}0.92 \\
(0.70,1.20) \\
\end{array}$ & $\begin{array}{l}1.09 \\
(0.81,1.46)\end{array}$ \\
\hline$+\mathrm{CRP}$ and IL-6 & $\begin{array}{l}1.14 \\
(1.04,1.25)\end{array}$ & $\begin{array}{l}1.21 \\
(0.95,1.55)\end{array}$ & $\begin{array}{l}1.02 \\
(0.79,1.55)\end{array}$ & $\begin{array}{l}1.34 \\
(1.03,1.75)\end{array}$ & $\begin{array}{l}1.05 \\
(0.95,1.17)\end{array}$ & $\begin{array}{l}0.96 \\
(0.75,1.23)\end{array}$ & $\begin{array}{l}0.91 \\
(0.69,1.20)\end{array}$ & $\begin{array}{l}1.09 \\
(0.82,1.47)\end{array}$ \\
\hline \multicolumn{9}{|c|}{$e G F R<60 \mathrm{ml} / \mathrm{min} / 1.73 \mathrm{~m}^{2}$} \\
\hline Subjects & 989 & 251 & 253 & 283 & 987 & 234 & 249 & 288 \\
\hline $\begin{array}{l}\text { Age, sex, race, baseline } \\
\text { eGFR-cysC }\end{array}$ & $\begin{array}{l}0.73 \\
(0.43,1.23)\end{array}$ & $\begin{array}{l}1.06 \\
(0.65,1.73)\end{array}$ & $\begin{array}{l}1.56 \\
(0.96,2.53)\end{array}$ & $\begin{array}{l}1.03 \\
(0.62,1.69)\end{array}$ & $\begin{array}{l}1.15 \\
(0.98,1.35)\end{array}$ & $\begin{array}{l}1.13 \\
(0.69,1.86)\end{array}$ & $\begin{array}{l}1.03 \\
(0.62,1.71)\end{array}$ & $\begin{array}{l}1.36 \\
(0.83,2.22)\end{array}$ \\
\hline+ Risk factors $^{\mathrm{b}}$ & $\begin{array}{l}0.80 \\
(0.46,1.40) \\
\end{array}$ & $\begin{array}{l}0.95 \\
(0.57,1.58) \\
\end{array}$ & $\begin{array}{l}1.48 \\
(0.89,2.44) \\
\end{array}$ & $\begin{array}{l}0.95 \\
(0.56,1.61)\end{array}$ & $\begin{array}{l}1.20 \\
(1.00,1.44) \\
\end{array}$ & $\begin{array}{l}1.21 \\
(0.72,2.02)\end{array}$ & $\begin{array}{l}1.03 \\
(0.60,0.79) \\
\end{array}$ & $\begin{array}{l}1.46 \\
(0.82,2.60) \\
\end{array}$ \\
\hline$+\mathrm{CRP}$ and IL-6 & $\begin{array}{l}0.74 \\
(0.42,1.33)\end{array}$ & $\begin{array}{l}0.94 \\
(0.56,1.56)\end{array}$ & $\begin{array}{l}1.50 \\
(0.91,2.48)\end{array}$ & $\begin{array}{l}0.94 \\
(0.55,1.59)\end{array}$ & $\begin{array}{l}1.23 \\
(1.02,1.47)\end{array}$ & $\begin{array}{l}1.24 \\
(0.74,2.07)\end{array}$ & $\begin{array}{l}1.05 \\
(0.61,1.8)\end{array}$ & $\begin{array}{l}1.56 \\
(0.87,2.79)\end{array}$ \\
\hline
\end{tabular}

Figures represent odds ratios $(95 \% \mathrm{CI})$, except where otherwise indicated. Rapid decline defined as: $\Delta \mathrm{eGFR}$-cysC $>3 \mathrm{ml} / \mathrm{min} / 1.73 \mathrm{~m}{ }^{2}$ per year. Baseline CKD defined by CKD-Epi eGFR $\geq$ or $<60 \mathrm{ml} / \mathrm{min} / 1.73 \mathrm{~m}^{2}$. Quartile 1 is the reference group. eGFR-cysC = Cystatin C-based eGFR.

${ }^{a}$ Quartile ranges for Lp-PLA 2 antigen: <257.31, 257.31-326.67, 326.68-404.68, >404.68; quartile ranges for Lp-PLA 2 activity: $<30.41,30.42-37.82$, $37.83-46.60,>46.60$.

${ }^{\mathrm{b}}$ Diabetes, systolic blood pressure, antihypertensive medications, LDL and HDL cholesterol, lipid-lowering medications, prevalent coronary heart disease.

Fig. 1. Association of Lp-PLA $\mathrm{A}_{2}$ antigen with kidney function decline (in $\mathrm{ml} /$ $\mathrm{min} / 1.73 \mathrm{~m}^{2}$ per year) among elderly persons by LDL, HDL and CRP levels at baseline.

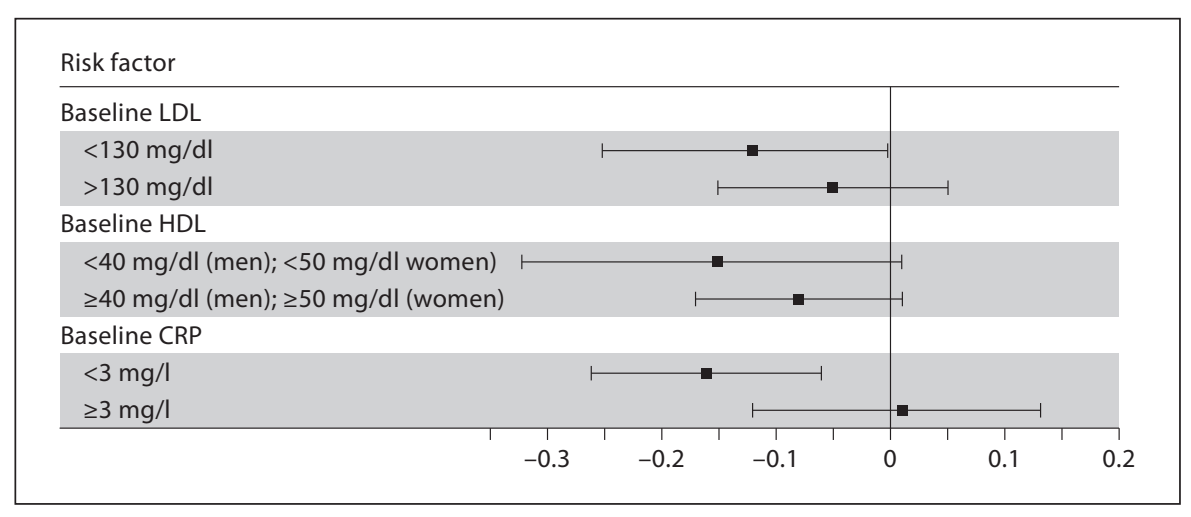

\section{Discussion}

In this study, we found that Lp-PLA ${ }_{2}$ antigen, not activity, was significantly associated with kidney function decline and rapid kidney function decline among elderly persons without CKD (eGFR $\geq 60 \mathrm{ml} / \mathrm{min} / 1.73 \mathrm{~m}^{2}$ ), independent of lipids, CRP and IL-6. Our findings have important implications for the understanding of potential pathways that may link CVD and kidney disease. Lp-
$\mathrm{PLA}_{2}$ has emerged as an independent risk factor for heart failure, stroke, coronary heart disease and cardiovascular death [8-10]. Lp-PLA ${ }_{2}$ appears to be associated with the development of endothelial dysfunction and inflammation and disruption of the arterial intima by hydrolyzing oxidized phospholipids and oxidating LDL [12-15]. Since several studies have shown that subclinical CVD [4], endothelial dysfunction [29], and reduced arterial elasticity [30] are associated with faster rates of kidney function 
decline, it is possible that Lp-PLA 2 is a novel common pathway of vascular injury in CKD and CVD.

Moreover, Lp-PLA 2 may be involved in an important pathway for development of CKD. In particular, our findings may shed light on why previous literature on the association of serum concentration of lipids and inflammatory markers with kidney disease has been inconsistent $[31,32]$. Lp-PLA $A_{2}$ travels bound preferentially to small LDL, and it hydrolyzes phospholipids, thus promoting atherogenic activity of LDL. Studying total LDL cholesterol and kidney disease may not fully capture these associations. In fact, in a study from the Multi-Ethnic Study of Atherosclerosis, levels of small LDL particles, but not conventional LDL levels were associated with early kidney dysfunction [33]. We found that inflammation did not attenuate our findings. Since the direction of the association between inflammation and kidney disease remains unclear $[5,6]$, future studies on the interplay between Lp-PLA 2 , inflammation, and kidney function decline are warranted.

We are the first to report an independent association between Lp-PLA $A_{2}$ antigen (mass) and kidney function decline. Strengths of this study include the well-characterized population, repeated measures of kidney function and the prospective design. Moreover, the use of cystatin $\mathrm{C}$ as a kidney function marker improves prediction of kidney decline in the elderly. However, we are limited by no direct measure of GFR. The small observed changes in eGFR may have reduced our ability to detect anything less than large associations. Future studies should include persons with large changes in eGFR over time [34].

However, no large, community-based epidemiological studies include iothalamate GFR. Our findings that Lp$\mathrm{PLA}_{2}$ antigen but not activity are associated with kidney function decline are in accordance with prior reports from CHS and a report on differing associations between antigen and activity and calcified coronary plaque in a young cohort [10,35]. These differences in associations between antigen and activity may be due to differences in assay design or variability. Alternatively, it is possible that the Lp-PLA $A_{2}$ antigen assay can better capture the complexity of the atherogenic process, particularly in this elderly cohort. Future studies are needed to elucidate these mechanisms. Our findings that the association of Lp$\mathrm{PLA}_{2}$ and kidney function decline was not observed among persons with eGFR $<60 \mathrm{ml} / \mathrm{min} / 1.73 \mathrm{~m}^{2}$ should be interpreted with caution, as it is possible that survival bias and regression to the mean may account for these findings. Alternatively, it is possible that certain pathways may have different relative importance in the development versus progression of CKD. We are unable to account for any possible associations with albuminuria, as CHS did not measure albuminuria at baseline.

In summary, we found that Lp-PLA 2 antigen (mass) is independently associated with kidney function decline and rapid kidney function decline among elderly persons with preserved GFR. Future studies should evaluate this novel pathway in other populations. Moreover, more studies are needed to elucidate causal pathways.

\section{Acknowledgments}

CHS is supported by contract numbers N01-HC-85079 through N01-HC-85086, N01-HC-35129, N01 HC-15103, N01 HC-55222, N01-HC-75150, N01-HC-45133, grant number U01 HL080295 from the National Heart, Lung, and Blood Institute, with additional contribution from the National Institute of Neurological Disorders and Stroke. This project is also supported by RO1 AG 027002 (Sarnak) and K23SK082793 (Peralta). A full list of principal CHS investigators and institutions can be found at http://www.chs-nhlbi.org/pi.htm.

\section{Disclosure Statement}

This study is supported by a grant from GlaxoSmithKline (GSK). Dr. Koro is an employee at GSK. Drs. Peralta, Cushman, and Fitzpatrick have funding from GSK. The funding sources did not have a role in the design, analysis or approval of the manuscript.

\section{References}

Lp-PLA 2 and Kidney Function Decline
The Health, Aging, and Body Composition Study. Arch Intern Med 2006;166:13961402 Hsu CY: Chronic kidney disease and the risks of death, cardiovascular events, and hospitalization. N Engl J Med 2004;351: 1296-1305.

2 Bibbins-Domingo K, Chertow GM, Fried LF, Odden MC, Newman AB, Kritchevsky SB, Harris TB, Satterfield S, Cummings SR, Shlipak MG: Renal function and heart failure risk in older black and white individuals:
3 Shlipak MG, Katz R, Sarnak MJ, Fried LF, Newman AB, Stehman-Breen C, Seliger SL, Kestenbaum B, Psaty B, Tracy RP, Siscovick DS: Cystatin C and prognosis for cardiovascular and kidney outcomes in elderly persons without chronic kidney disease. Ann Intern Med 2006;145:237-246. 
-4 Shlipak MG, Katz R, Kestenbaum B, Fried LF, Siscovick D, Sarnak MJ: Clinical and subclinical cardiovascular disease and kidney function decline in the elderly. Atherosclerosis 2009;204:298-303.

5 Keller C, Katz R, Sarnak MJ, Fried LF, Kestenbaum B, Cushman M, Shlipak MG: Inflammatory biomarkers and decline in kidney function in the elderly: The Cardiovascular Health Study. Nephrol Dial Transplant 2010;25:119-124.

-6 Dubin R, Cushman M, Folsom AR, Fried LF, Palmas W, Peralta CA, Wassel C, Shlipak MG: Kidney function and multiple hemostatic markers: cross sectional associations in the multi-ethnic study of atherosclerosis. BMC Nephrol 2011;12:3.

-7 Upadhyay A, Larson MG, Guo CY, Vasan RS, Lipinska I, O’Donnell CJ, Kathiresan S, Meigs JB, Keaney JF Jr, Rong J, Benjamin EJ, Fox CS: Inflammation, kidney function and albuminuria in the Framingham Offspring Cohort. Nephrol Dial Transplant 2011;26: 920-926.

-8 Association of estimated glomerular filtration rate and albuminuria with all-cause and cardiovascular mortality in general population cohorts: a collaborative meta-analysis. Lancet 2010;375:2073-2081.

-9 Packard CJ, O'Reilly DS, Caslake MJ, McMahon $\mathrm{AD}$, Ford I, Cooney J, Macphee $\mathrm{CH}$, Suckling KE, Krishna M, Wilkinson FE, Rumley A, Lowe GD: Lipoprotein-associated phospholipase A2 as an independent predictor of coronary heart disease. West of Scotland coronary prevention study group. $\mathrm{N}$ Engl J Med 2000;343:1148-1155.

10 Suzuki T, Solomon C, Jenny NS, Tracy R, Nelson JJ, Psaty BM, Furberg C, Cushman M: Lipoprotein-associated phospholipase $\mathrm{A}(2)$ and risk of congestive heart failure in older adults: The Cardiovascular Health Study. Circ Heart Fail 2009;2:429-436.

- 11 Thompson A, Gao P, Orfei L, Watson S, Di Angelantonio E, Kaptoge S, Ballantyne C, Cannon CP, Criqui M, Cushman M, Hofman A, Packard C, Thompson SG, Collins R, Danesh J: Lipoprotein-associated phospholipase $A(2)$ and risk of coronary disease, stroke, and mortality: collaborative analysis of 32 prospective studies. Lancet 2010;375: 1536-1544.

12 Stafforini DM, Tjoelker LW, McCormick SP, Vaitkus D, McIntyre TM, Gray PW, Young SG, Prescott SM: Molecular basis of the interaction between plasma platelet-activating factor acetylhydrolase and low density lipoprotein. J Biol Chem 1999;274:7018-7024.

$\checkmark 13$ Corson MA: Phospholipase A2 inhibitors in atherosclerosis: The race is on. Lancet 2009; 373:608-610.

14 MacPhee CH, Moores KE, Boyd HF, Dhanak D, Ife RJ, Leach CA, Leake DS, Milliner KJ, Patterson RA, Suckling KE, Tew DG, Hickey DM: Lipoprotein-associated phospholipase A2, platelet-activating factor acetylhydro- lase, generates two bioactive products during the oxidation of low-density lipoprotein: Use of a novel inhibitor. Biochem J 1999;338: 479-487.

15 Zalewski A, Macphee C: Role of lipoproteinassociated phospholipase A2 in atherosclerosis: Biology, epidemiology, and possible therapeutic target. Arterioscler Thromb Vasc Biol 2005;25:923-931.

16 Dubin R, Shlipak M, Li Y, Ix J, de Boer IH, Jenny N, Peralta CA: Racial differences in the association of pentraxin-3 with kidney dysfunction: the Multi-Ethnic Study of Atherosclerosis. Nephrol Dial Transplant 2011;26: 1903-1908.

17 Peralta CA, Adeney KL, Shlipak MG, Jacobs D Jr, Duprez D, Bluemke D, Polak J, Psaty B, Kestenbaum BR: Structural and functional vascular alterations and incident hypertension in normotensive adults: the Multi-Ethnic Study of Atherosclerosis. Am J Epidemiol 2010;171:63-71.

18 Fried LP, Borhani NO, Enright P, Furberg CD, Gardin JM, Kronmal RA, Kuller LH, Manolio TA, Mittelmark MB, Newman A, et al: The Cardiovascular Health Study: design and rationale. Ann Epidemiol 1991;1:263276.

19 Levey AS, Stevens LA, Schmid CH, Zhang YL, Castro AF, 3rd, Feldman HI, Kusek JW, Eggers P, Van Lente F, Greene T, Coresh J: A new equation to estimate glomerular filtration rate. Ann Intern Med 2009;150:604-612.

20 Stevens LA, Coresh J, Schmid CH, Feldman HI, Froissart M, Kusek J, Rossert J, Van Lente F, Bruce RD 3rd, Zhang YL, Greene T, Levey AS: Estimating GFR using serum cystatin C alone and in combination with serum creatinine: a pooled analysis of 3,418 individuals with CKD. Am J Kidney Dis 2008;51: 395-406

21 Rifkin DE, Shlipak MG, Katz R, Fried LF, Siscovick D, Chonchol M, Newman AB, Sarnak MJ: Rapid kidney function decline and mortality risk in older adults. Arch Intern Med 2008;168:2212-2218.

22 Kdoqi clinical practice guidelines and clinical practice recommendations for diabetes and chronic kidney disease. Am J Kidney Dis 2007;49:S12-S154

23 Perkins BA, Nelson RG, Ostrander BE, Blouch KL, Krolewski AS, Myers BD, Warram JH: Detection of renal function decline in patients with diabetes and normal or elevated GFR by serial measurements of serum cystatin $\mathrm{C}$ concentration: results of a 4-year follow-up study. J Am Soc Nephrol 2005; 16 : 1404-1412.

24 Sarnak MJ, Katz R, Fried LF, Siscovick D, Kestenbaum B, Seliger S, Rifkin D, Tracy R, Newman AB, Shlipak MG: Cystatin C and aging success. Arch Intern Med 2008;168: 147-153.

25 Shlipak MG, Katz R, Kestenbaum B, Fried LF, Newman AB, Siscovick DS, Stevens L, Sarnak MJ: Rate of kidney function decline in older adults: a comparison using creatinine and cystatin C. Am J Nephrol 2009;30: 171-178.

-26 Peralta CA, Katz R, Sarnak MJ, Ix J, Fried LF, De Boer I, Palmas W, Siscovick D, Levey AS, Shlipak MG: Cystatin C identifies chronic kidney disease patients at higher risk for complications. J Am Soc Nephrol 2011;22: 147-155.

27 Cushman M, Cornell ES, Howard PR, Bovill EG, Tracy RP: Laboratory methods and quality assurance in the cardiovascular health study. Clin Chem 1995;41:264-270.

28 Ballantyne CM, Hoogeveen RC, Bang H, Coresh J, Folsom AR, Heiss G, Sharrett AR: Lipoprotein-associated phospholipase A2, high-sensitivity C-reactive protein, and risk for incident coronary heart disease in middle-aged men and women in the atherosclerosis risk in communities (ARIC) study. Circulation 2004;109:837-842.

29 Perticone F, Maio R, Perticone M, Sciacqua A, Shehaj E, Naccarato P, Sesti G: Endothelial dysfunction and subsequent decline in glomerular filtration rate in hypertensive patients. Circulation 2010;122:379-384.

30 Peralta CA, Jacob DR Jr, Katz R, Ix J, Madero M, Duprez D, Sarnak M, Criqui M, Kramer H, Palmas W, Herrington D, Shlipak M: The association of pulse pressure, arterial elasticity and endothelial function with kidney function decline among adults without chronic kidney disease: The Multi-Ethnic Study of Atherosclerosis. Am J Kidney Dis, in press.

31 Hunsicker LG, Adler S, Caggiula A, England BK, Greene T, Kusek JW, Rogers NL, Teschan PE: Predictors of the progression of renal disease in the modification of diet in renal disease study. Kidney Int 1997;51:1908-1919.

- 32 Muntner P, Coresh J, Smith JC, Eckfeldt J, Klag MJ: Plasma lipids and risk of developing renal dysfunction: The Atherosclerosis Risk in Communities Study. Kidney Int 2000;58: 293-301.

-33 de Boer IH, Kestenbaum B, Shoben AB, Michos ED, Sarnak MJ, Siscovick DS: 25-hydroxyvitamin D levels inversely associate with risk for developing coronary artery calcification. J Am Soc Nephrol 2009;20:18051812.

34 Madder RD, Hickman L, Crimmins GM, Puri M, Marinescu V, McCullough PA, Safian RD: Validity of estimated glomerular filtration rates for assessment of baseline and serial renal function in patients with atherosclerotic renal artery stenosis: implications for clinical trials of renal revascularization. Circ Cardiovasc Interv 2011;4:219-225.

- 35 Iribarren C, Gross MD, Darbinian JA, Jacobs DR Jr, Sidney S, Loria CM: Association of lipoprotein-associated phospholipase A2 mass and activity with calcified coronary plaque in young adults: The CARDIA study. Arterioscler Thromb Vasc Biol 2005;25:216221. 